\title{
The Tractability Frontier for NFA Minimization^
}

\author{
Henrik Björklund and Wim Martens \\ TU Dortmund \\ henrik.bjoerklund@udo.edu \\ wim.martens@udo.edu
}

\begin{abstract}
We essentially show that minimizing finite automata is NPhard as soon as one deviates from the class of deterministic finite automata. More specifically, we show that minimization is NP-hard for all finite automata classes that subsume the class that is unambiguous, allows at most one state $q$ with a non-deterministic transition for at most one alphabet symbol $a$, and is allowed to visit state $q$ at most once in a run. Furthermore, this result holds even for automata that only accept finite languages.
\end{abstract}

\section{Introduction}

The regular languages are immensely important, not only in theoretical computer science, but also in practical applications. When using regular languages in practice, the developer is often faced with a trade-off between the descriptive complexity and the complexity of optimization. Concretely, it has been known for a long time that there are regular languages for which non-deterministic finite automata (NFAs) can provide an exponentially more succinct description than deterministic finite automata (DFAs) [13]. On the other hand, many decision problems that are solvable in polynomial time for DFAs, i.e., equivalence, inclusion, and universality, are computationally hard for NFAs.

The choice of a representation mechanism can therefore be crucial. If the set of regular languages used in an application is relatively constant, membership tests are the main language operations, and economy of space is an issue, NFAs are probably the right choice. If, on the other hand, the languages change frequently, and inclusion or equivalence tests are frequent, DFAs may be more attractive.

Since both NFAs and DFAs have their disadvantages, a lot of effort has been spent on trying to find intermediate models, i.e., finite automata that have some limited form of non-determinism. A rather successful intermediate model is the class of unambiguous finite automata (UFAs). ${ }^{1}$ While in general still being exponentially more succinct than an equivalent DFA for the same language, static analysis questions such as inclusion and equivalence can be solved in PTIME for UFAs [17]. However, UFAs do not allow for tractable state minimization [11].

\footnotetext{
* This work was supported by the DFG Grant SCHW678/3-1.

${ }^{1}$ An automaton is unambiguous if it has at most one accepting run for each word.
} 
Therefore, the question whether there are good intermediate models between DFAs and NFAs needs to be revisited for state minimization. The main result of this paper is that, probably, no such good models exist. Even the tiniest bit of non-determinism makes the minimization problem NP-hard.

Every undergraduate computer science curriculum teaches its students how to minimize a DFA in polynomial time. In contrast, minimizing unrestricted NFAs is PSPACE-complete [18]. The minimization problem for automata with varying degrees of non-determinism was studied in a seminal paper by Jiang and Ravikumar in 1993 [11]. Among other results, they thoroughly investigated the minimization problem for UFAs. They showed the following.

- Given a UFA, finding the minimal equivalent UFA is NP-complete.

- Given a DFA, finding the minimal equivalent UFA is NP-complete.

- Given a DFA, finding the minimal equivalent NFA is PSPACE-complete.

Minimization problems have even been studied for automata with unary alphabets; see, e.g., $[10,5]$.

Recently, Malcher [12] improved on the results of Jiang and Ravikumar in the sense that he showed that finite automata with quite a small amount of non-determinism are hard to minimize. More precisely, he showed the following:

(a) Minimization is NP-complete for automata that can non-deterministically choose between a fixed number of initial states, but are otherwise deterministic.

(b) Minimization is NP-complete for non-deterministic automata with a constant number of computations for each string. ${ }^{2}$

Whereas Malcher made significant progress in showing that minimization is hard for non-deterministic automata, he was not yet able to answer the entire question. Therefore, he asks whether there are relaxations of the deterministic automata model at all for which minimization is tractable as an important open problem. In this respect, he mentions the class of automata with at most two computations for each string and the two classes (a) and (b) above with the added restriction of unambiguousness as important remaining cases. In the present paper, we settle these open questions and provide a uniform NP-hardness proof for all classes of automata mentioned above.

In brief, we define a class $\delta$ NFA of automata that are unambiguous, have at most two computations per string, and have at most one state $q$ with two outgoing $a$-transitions, for at most one symbol $a$. Then, we show that minimization is NP-hard for all classes of finite automata that include $\delta$ NFAs, and show that these hardness results can also be adapted to the setting of unambiguous automata that can non-deterministically choose between two start states, but are deterministic everywhere else. This solves the open cases mentioned by Malcher. On the other hand, there are relaxations of the deterministic automaton model that allow tractable minimization. We show that, if we add to the definition of

\footnotetext{
${ }^{2}$ Actually, he showed this for automata with constant branching, which is slightly different from the number of computations; see Section 2.1.
} 
$\delta$ NFAs that each word should have at most one rejecting computation, minimization becomes tractable again. However, the minimal automata in this class are the DFAs, so this class is not likely to be very useful in practice.

Other related work. An overview of state and transition complexity of NFAs can be found in [15]. Known results about the trade-off between amount of non-determinism and descriptional complexity are surveyed in [2]. The problems of producing small NFAs from regular expressions has been considered in $[9$, 16]. There has also been some work on approximating minimal NFAs $[3,4,6]$, basically showing that approximation is very hard. The work of Hromkovic et al. [8] about measuring non-determinism in finite automata will be relevant to us in Section 2. Since the minimization problem for NFAs is hard, the bisimulation minimization problem has also been considered; see, e.g., [14,1].

Due to space restrictions, some proofs and parts of proofs have been omitted, and will appear in the full version of the paper.

\section{Preliminaries}

By $\Sigma$ we always denote a finite alphabet. A (non-deterministic) finite automaton (NFA) over $\Sigma$ is a tuple $A=(\operatorname{States}(A), \operatorname{Alpha}(A), \operatorname{Rules}(A), \operatorname{init}(A), \operatorname{Final}(A))$, where $\operatorname{States}(A)$ is its finite set of states, $\operatorname{Alpha}(A)=\Sigma, \operatorname{init}(A) \in \operatorname{States}(A)$ is its initial state, $\operatorname{Final}(A) \subseteq \operatorname{States}(A)$ is its set of final states, and $\operatorname{Rules}(A)$ is a set of transition rules of the form $q_{1} \stackrel{a}{\rightarrow} q_{2}$, where $q_{1}, q_{2} \in \operatorname{States}(A)$ and $a \in \Sigma$. The size of an automaton is $|\operatorname{States}(A)|$, i.e., its number of states. A finite automaton is deterministic if, for each $q_{1} \in \operatorname{States}(A)$ and $a \in \operatorname{Alpha}(A)$, there is at most one $q_{2} \in \operatorname{States}(A)$ such that $q_{1} \stackrel{a}{\rightarrow} q_{2} \in \operatorname{Rules}(A)$. By DFA we denote the class of deterministic finite automata.

A run, or computation, $r$ of $A$ on a word $w=a_{1} \cdots a_{n} \in \Sigma^{*}$ is a string $q_{1} \cdots q_{n} \in \operatorname{States}(A)^{*}$ such that $\operatorname{init}(A) \stackrel{a_{1}}{\rightarrow} q_{1} \in \operatorname{Rules}(A)$ and, for each $i=$ $1, \ldots, n-1, q_{i} \stackrel{a_{i+1}}{\longrightarrow} q_{i+1} \in \operatorname{Rules}(A)$. The run is accepting if $q_{n} \in \operatorname{Final}(A)$. The language of $A$ is the set of words $w$ such that there exists an accepting run of $A$ on $w$. A finite automaton $A$ is unambiguous if, for each string $w$, there exists at most one accepting run of $A$ on $w$.

Let $\mathcal{N}_{1}$ and $\mathcal{N}_{2}$ be two classes of NFAs. We say that $\mathcal{N}_{1} \subseteq \mathcal{N}_{2}$ if each automaton in $\mathcal{N}_{1}$ also belongs to $\mathcal{N}_{2}$. For example, DFA $\subseteq$ NFA.

\subsection{Notions of Non-Determinism}

We recall some standard measures of non-determinism in a finite automaton. For a state $q$ and an alphabet symbol $a$, the degree of non-determinism of a pair $(q, a)$, denoted by $\operatorname{degree}(q, a)$ is the number $k$ of different states $q_{1}, \ldots, q_{k}$ such that, for all $1 \leq i \leq k, q \stackrel{a}{\rightarrow} q_{i} \in \operatorname{Rules}(A)$. We say that $A$ has degree of non-determinism $k$, denoted by $\operatorname{degree}(A)=k$, if $\operatorname{degree}(q, a) \leq k$ for every $(q, a) \in \operatorname{States}(A) \times \operatorname{Alpha}(A)$, and there is at least one pair $(q, a)$ such that $\operatorname{degree}(q, a)=k$. 
The branching of an automaton is intuitively defined as the maximum product of the degrees of non-determinism over states in a possible run. Formally, the branching of $A$ on a word $w=a_{1} \cdots a_{n}$ is $\operatorname{branch}_{A}(w)=\max \left\{\Pi_{i=1}^{n} \operatorname{degree}\left(q_{i-1}\right.\right.$, $\left.a_{i}\right) \mid q_{1} \cdots q_{n}$ is a run of $A$ on $a_{1} \cdots a_{n}$, and $\left.\operatorname{init}(A)=q_{0}\right\}$. The branching of $A$, denoted $\operatorname{branch}(A)$, is $\max \left\{\operatorname{branch}_{A}(w) \mid w \in L(A)\right\}$ if this quantity is defined, and otherwise $\infty$.

Hromkovic et al. [8] define three measures non-determinism for a finite automaton $A$ : advice $(A)$, computations $(A)$, and ambig $(A)$. These measures are defined as follows: advice $(A)$ is the maximum number of non-deterministic choices during any computation of $A$, $\operatorname{computations}(A)$ is the maximum number of different computations of $A$ on any word, ${ }^{3}$ and $\operatorname{ambig}(A)$ is the maximum number of different accepting computation of $A$ on any word. For the formal definitions of these concepts, we refer to [8].

\subsection{A Notion of Very Little Non-Determinism}

Next we define the notion of a $\delta$ NFA. The intuition is that such an automaton should allow only a very small amount of non-determinism.

Definition 1. A $\delta N F A$ is an NFA $A$ with the following properties

- $A$ is unambiguous;

$-\operatorname{branch}(A) \leq 2$; and

- there is at most one pair $(q, a)$ such that degree $(q, a)=2$.

For $\delta$ NFAs, we have that $\operatorname{degree}(A) \leq 2, \operatorname{advice}(A) \leq 1, \operatorname{computations}(A) \leq 2$, and $\operatorname{ambig}(A)=1$. Notice that any one of $\operatorname{degree}(A)=1$, $\operatorname{advice}(A)=0$, or $\operatorname{computations}(A)=1 \mathrm{implies}$ that $A$ is deterministic. Also, $\operatorname{ambig}(A)=1$ is the minimum value possible for any automaton that accepts at least one string.

\subsection{The Minimization Problem}

We define the minimization problem in two flavors. For two classes of finite automata $\mathcal{N}_{1}$ and $\mathcal{N}_{2}$ the $\mathcal{N}_{1} \rightarrow \mathcal{N}_{2}$ minimization problem is the following problem. Given a finite automaton $A$ in $\mathcal{N}_{1}$ and an integer $k$, does there exist a finite automaton $B$ in $\mathcal{N}_{2}$ of size at most $k$ such that $L(A)=L(B)$ ? For a class $\mathcal{N}$ of finite automata, the minimization problem for $\mathcal{N}$ is then simply the $\mathcal{N} \rightarrow \mathcal{N}$ minimization problem.

Suppose that the $\mathcal{N}_{1} \rightarrow \mathcal{N}_{2}$ minimization problem is hard for a complexity class $C$, and let $\mathcal{N}_{3}$ be a class of automata such that $\mathcal{N}_{1} \subseteq \mathcal{N}_{3}$. Then the $\mathcal{N}_{3} \rightarrow \mathcal{N}_{2}$ minimization problem is also trivially hard for $C$. However, assuming that $\mathcal{N}_{1} \rightarrow \mathcal{N}_{2}$ is hard for $C$ and that $\mathcal{N}_{2} \subseteq \mathcal{N}_{3}$, there is, as far as we know, no general argument that also makes the $\mathcal{N}_{1} \rightarrow \mathcal{N}_{3}$ minimization problem hard for $C$, as finding a small $\mathcal{N}_{3}$ automaton might be easier than finding a small $\mathcal{N}_{2}$

\footnotetext{
${ }^{3}$ Hromkovic et al. wrote leaf $(A)$ instead of computations $(A)$.
} 
automaton in general. ${ }^{4}$ Therefore, we will prove directly that minimization is NP-hard for all classes of automata between $\delta$ NFAs and NFAs.

\subsection{Are $\delta$ NFAs the Closest Possible to Determinism?}

Before we give more intuition about this question, we first note that there are in fact two incomparable notions of determinism for finite automata: determinism and reverse determinism. ${ }^{5}$ Both classes can be efficiently minimized by the same algorithm, modulo a simple pre- and post-processing step for reverse deterministic automata. We view these two classes as the two possible "optima" in the spectrum of determinism, as they arise very naturally from the fact that one can either read strings from left to right or from right to left. From now on, we only consider the proximity of $\delta$ NFAs to (left-to-right deterministic) DFAs.

We believe that one can always think of more and more exotic notions of non-determinism that come closer and closer to DFAs. ${ }^{6}$ We provide an example here. Define the class $\mathcal{C}$ to be the class of $\delta$ NFAs with the additional condition that, for each word $w$, there can be at most one rejecting computation of $A$ on $w$. (Thus, for each $w$, there can be at most two runs - one accepting and one rejecting.) This notion of non-determinism lies strictly between DFAs and $\delta$ NFAs $(\mathrm{DFA} \subseteq \mathcal{C} \subseteq \delta \mathrm{NFA})$.

Consider the minimization problem for $\mathcal{C}$ and let $A$ be an arbitrary automaton in $\mathcal{C}$. We will argue that the minimal $\mathcal{C}$-automaton for $L(A)$ is a DFA. Suppose that $A$ is not a DFA. Let $q$ and $a$ be the unique state and label so that degree $(q, a)=2$. Let $q_{1}$ and $q_{2}$ be the two states such that $q \stackrel{a}{\rightarrow} q_{1}$ and $q \stackrel{a}{\rightarrow} q_{2}$ are in Rules $(A)$. Let $w$ be an arbitrary string that leads $A$ to state $q$. By definition of $\mathcal{C}, A$ must accept every string of the form $w a w^{\prime}$, where $w^{\prime}$ is an arbitrary word in $\operatorname{Alpha}(A)^{*}$. (If waw' would be rejected, then there would be two rejecting runs, one over $q_{1}$ and one over $q_{2}$.) Therefore, we can make $A$ strictly smaller by merging the two states $q_{1}, q_{2}$ to $q_{3}$, removing all outgoing transitions from $q_{3}$, making $q_{3}$ a final state, and adding loop transitions from $q_{3}$ to itself for each alphabet symbol. Moreover, by this operation, $A$ becomes deterministic. Hence, every automaton $A$ in $\mathcal{C}$ that is not a DFA can be rewritten as a smaller DFA. This means that, in the class $\mathcal{C}$, the minimal automata are the DFAs. In particular, this also puts the minimization problem for $\mathcal{C}$ into PTIME.

From the above it is clear that $\delta$ NFAs are certainly not the closest possible to determinism that one can get. Rather, it is the closest class to DFAs we were able to find that takes advantage of the succinctness of nondeterminism in a nontrivial way.

Our NP-hardness result for the minimization of $\delta$ NFAs therefore puts the tractability frontier precisely between $\delta$ NFAs and the above mentioned class $\mathcal{C}$; two classes that are extremely close to one another.

\footnotetext{
${ }^{4}$ This is also why, e.g., Malcher explicitly proves NP-hardness for minimizing various classes of automata that are included in one another (Lemmas 3 and 11 in [12]).

${ }^{5}$ The latter is the class for which the inverted transitions are deterministic.

${ }^{6}$ One could, for instance, take the class of DFAs and add a single NFA.
} 


\section{Minimizing Non-Deterministic Automata is Hard}

The main result of this section is the following

Theorem 2. Let $\mathcal{N}$ be a class of finite automata. If $\delta N F A \subseteq \mathcal{N}$ then $D F A \rightarrow \mathcal{N}$ minimization is NP-hard.

Corollary 3. For each class $\mathcal{N}$ of finite automata such that $\delta N F A \subseteq \mathcal{N}$, the minimization problem for $\mathcal{N}$ is NP-hard.

We start by formally defining the decision problems that are of interest to us, and then sketch an intuitive overview of our proof. Given an undirected graph $G=(V, E)$ such that $V$ is its set of vertices and $E \subseteq V \times V$ is its set of edges, we say that a set of vertices $V C \subseteq V$ is a vertex cover of $G$ if, for every edge $\left(v_{1}, v_{2}\right) \in E, V C$ contains $v_{1}, v_{2}$, or both.

If $B$ and $C$ are finite collections of finite sets, we say that $B$ is a set basis for $C$ if, for each $c \in C$, there is a subcollection $B_{c}$ of $B$ whose union is $c$. We say that $B$ is a normal set basis for $C$ if, for each $c \in C$, there is a pairwise disjoint subcollection $B_{c}$ of $B$ whose union is $c$. We say that $B$ is a separable normal set basis for $C$ if $B$ is a normal set basis for $C$ and $B$ can be written as a disjoint union $B_{1} \uplus B_{2}$ such that, for each $c \in C$, the subcollection $B_{c}$ of $B$ contains at most one element from $B_{1}$ and at most one from $B_{2}$.

The following decision problems are considered in this paper. Vertex Cover asks, given a pair $(G, k)$ where $G$ is a graph and $k$ is an integer, whether there exists a vertex cover of $G$ of size at most $k$. Set Basis, Normal Set Basis, and Separable Normal Set Basis ask, given a pair $(C, s)$ where $C$ is a finite collection of finite sets and $s$ is an integer, whether there exists a set basis, resp., normal set basis, resp., separable normal set basis for $C$ containing at most $s$ sets.

The proof of Theorem 2 proceeds in several steps. First, we provide a slightly modified version of a known reduction from Vertex Cover to Normal Set Basis (Lemma 4 in [11]), showing that the latter problem is NP-hard. Second, we proceed to show that the set I of instances of Normal Set Basis obtained through this reduction has a number of interesting properties (Lemma 5). In particular, we show that if such an instance has a set basis of a certain size $s$, then it also has a normal set basis of size $s$. Third, we show that the the Normal Set Basis problem, for instances in $\mathbf{I}$ reduces to minimization for $\delta$ NFAs (Lemma 6 ).

The statement of Theorem 2 says that given a DFA, finding the minimal equivalent automaton in class $\mathcal{N}$ is NP-hard, for any class of finite automata that contains the $\delta$ NFAs. As argued in Section 2.3, using a DFA instead of a $\delta$ NFA as input of the problem strengthens the statement. Also, showing that $\mathrm{DFA} \rightarrow \delta \mathrm{NFA}$ is NP-hard doesn't immediately imply that DFA $\rightarrow \mathcal{N}$ is hard for every $\mathcal{N}$ that contains all $\delta$ NFAs. To show that this is actually the case, we prove that for the languages obtained in our reduction, the minimal NFAs are precisely one state smaller than the minimal $\delta$ NFAs (Lemma 6). For these languages, the minimization problem for $\delta$ NFAs and for NFAs is essentially the same problem.

We revisit a slightly modified reduction which is due to Jiang and Ravikumar [11], as our further results rely on a construction in their proof. 


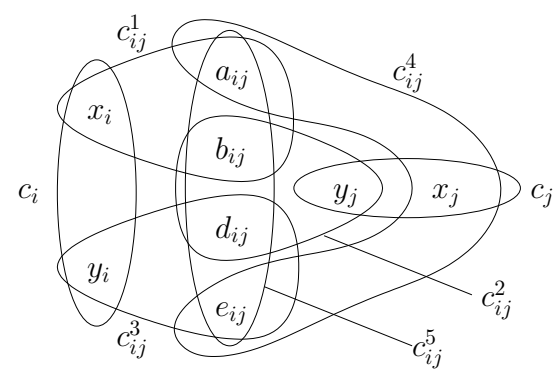

Fig. 1. The constructed sets $c_{i}, c_{j}, c_{i j}^{1}, \ldots, c_{i j}^{5}$ in the proof of Lemma 4 .

Lemma 4 (Jiang and Ravikumar [11]). Normal Set Basis is NP-complete.

Proof (Sketch). Obviously, Normal Set Basis is in NP. Indeed, given an input $(C, s)$ for Normal Set Basis, the NP algorithm simply guesses a collection $B$ containing at most $s$ sets, guesses the subcollections $B_{c}$ for each $c \in C$, and verifies whether the sets $B_{c}$ satisfy the necessary conditions.

We give a reduction from Vertex Cover to Normal Set Basis but omit the correctness proof. Given an input $(G, k)$ of Vertex Cover, where $G=(V, E)$ is a graph and $k$ is an integer, we construct in LOGSPACE an input $(C, s)$ of Normal Set Basis, where $C$ is a finite collection of finite sets and $s$ is an integer. In particular, $(C, s)$ is constructed such that $G$ has a vertex cover of size at most $k$ if and only if $C$ has a normal set basis containing at most $s$ sets.

For a technical reason which will become clear in later proofs, we assume without loss of generality that $k<|E|-3$. Notice that, under this restriction, Vertex Cover is still NP-complete under LOGSPACE reductions. Indeed, if $k \geq$ $|E|-3$, Vertex Cover can be solved in LOGSPACE by testing all possibilities of the at most 3 vertices which are not in the vertex cover, and verifying that there does not exist an edge between 2 of these 3 vertices.

Formally, let $V=\left\{v_{1}, \ldots, v_{n}\right\}$. For each $i=1, \ldots, n$, define $c_{i}$ to be the set $\left\{x_{i}, y_{i}\right\}$ which intuitively corresponds to the node $v_{i}$. Let $\left(v_{i}, v_{j}\right)$ be in $E$ with $i<j$. To each such edge we associate five sets as follows:

$$
\begin{array}{ll}
c_{i j}^{1}:=\left\{x_{i}, a_{i j}, b_{i j}\right\}, & c_{i j}^{4}:=\left\{x_{j}, a_{i j}, e_{i j}\right\}, \text { and } \\
c_{i j}^{2}:=\left\{y_{j}, b_{i j}, d_{i j}\right\}, & c_{i j}^{5}:=\left\{a_{i j}, b_{i j}, d_{i j}, e_{i j}\right\} . \\
c_{i j}^{3}:=\left\{y_{i}, d_{i j}, e_{i j}\right\}, &
\end{array}
$$

Figure 1 contains a graphical representation of the constructed sets $c_{i}, c_{j}, c_{i j}^{1}, \ldots$, $c_{i j}^{5}$ for some $\left(v_{i}, v_{j}\right) \in E$. Then, define

$$
C:=\left\{c_{i} \mid 1 \leq i \leq n\right\} \cup\left\{c_{i j}^{t} \mid\left(v_{i}, v_{j}\right) \in E, i<j, \text { and } 1 \leq t \leq 5\right\}
$$

and $s:=n+4|E|+k$. Notice that the collection $C$ contains $n+5|E|$ sets. Obviously, $C$ and $s$ can be constructed from $G$ and $k$ in LOGSPACE. 
We show that the reduction is also correct, that is, that $G$ has a vertex cover of size at most $k$ if and only if $C$ has a (separable) normal set basis containing at most $s$ sets.

$(\Rightarrow)$ : Let $G$ have a vertex cover $V C$ of size $k$. We need to show that $C$ has a normal set basis $B$ containing at most $s=n+4|E|+k$ sets.

To this end, we define a collection $B$ of sets as follows. For every $v_{i} \in V$,

- if $v_{i} \in V C$, we include both $\left\{x_{i}\right\}$ and $\left\{y_{i}\right\}$ in $B$;

- otherwise, we include $c_{i}=\left\{x_{i}, y_{i}\right\}$ in $B$.

The number of sets included in $B$ so far is $2 k+(n-k)=k+n$. Let $e=\left(v_{i}, v_{j}\right)$ (where $i<j$ ) be an arbitrary edge in $G$. Since $V C$ is a vertex cover, either $v_{i}$ or $v_{j}$ (or both) is in $V C$. When $v_{i}$ is in $V C$, we additionally include the sets

$$
\begin{array}{ll}
r_{i j}^{1}: & =\left\{a_{i j}, b_{i j}\right\}, \quad r_{i j}^{2}:=\left\{d_{i j}, e_{i j}\right\}, \\
r_{i j}^{3}:=\left\{y_{j}, b_{i j}, d_{i j}\right\}, \text { and } r_{i j}^{4}:=\left\{x_{j}, a_{i j}, e_{i j}\right\}
\end{array}
$$

in $B$. When $v_{i}$ is not in $V C$, we additionally include the sets

$$
\begin{aligned}
& r_{i j}^{5}:=\left\{a_{i j}, e_{i j}\right\}, \quad r_{i j}^{6}:=\left\{b_{i j}, d_{i j}\right\}, \\
& r_{i j}^{7}:=\left\{x_{i}, a_{i j}, b_{i j}\right\}, \text { and } r_{i j}^{8}:=\left\{y_{i}, d_{i j}, e_{i j}\right\}
\end{aligned}
$$

in $B$. This completes the definition of $B$. Notice that, when $v_{i} \in V C, c_{i j}^{1}, c_{i j}^{3}$, and $c_{i j}^{5}$ can be expressed as a disjoint union of members of $B$ as

$$
c_{i j}^{1}=\left\{x_{i}\right\} \uplus r_{i j}^{1}, \quad c_{i j}^{3}=\left\{y_{i}\right\} \uplus r_{i j}^{2}, \quad c_{i j}^{5}=r_{i j}^{1} \uplus r_{i j}^{2}
$$

and that $c_{i j}^{2}=r_{i j}^{3}$ and $c_{i j}^{4}=r_{i j}^{4}$ are members of $B$. Analogously, when $v_{i} \notin V C$, $c_{i j}^{2}, c_{i j}^{4}$, and $c_{i j}^{5}$ can be expressed as a disjoint union of members of $B$ as

$$
c_{i j}^{2}=\left\{y_{j}\right\} \uplus r_{i j}^{6}, \quad c_{i j}^{4}=\left\{x_{j}\right\} \uplus r_{i j}^{5}, \quad c_{i j}^{5}=r_{i j}^{5} \uplus r_{i j}^{6}
$$

and $c_{i j}^{1}=r_{i j}^{7}$ and $c_{i j}^{3}=r_{i j}^{8}$ are members of $B$. Since the total number of sets included in $B$ for each edge is four, $B$ contains $(k+n)+4|E|=s$ sets. From the above argument it is also obvious that $B$ is a normal set basis for $C$.

Notice that $B$ is in fact a separable normal set basis for $C$. Indeed, we can partition $B$ into the sets

$$
\begin{aligned}
& B_{1}=\left\{\left\{x_{i}\right\},\left\{x_{j}, y_{j}\right\} \mid v_{i} \in V C, v_{j} \notin V C\right\} \\
& \cup\left\{r_{i j}^{2}, r_{i j}^{3} \mid\left(v_{i}, v_{j}\right) \in E, i<j, v_{i} \in V C\right\} \\
& \cup\left\{r_{i j}^{6}, r_{i j}^{7} \mid\left(v_{i}, v_{j}\right) \in E, i<j, v_{i} \notin V C\right\}
\end{aligned}
$$

and

$$
\begin{aligned}
B_{2}=\left\{\left\{y_{i}\right\} \mid v_{i} \in\right. & V C\} \\
\cup\left\{r_{i j}^{1}, r_{i j}^{4} \mid\left(v_{i}, v_{j}\right)\right. & \left.\in E, i<j, v_{i} \in V C\right\} \\
\cup & \left\{r_{i j}^{5}, r_{i j}^{8} \mid\left(v_{i}, v_{j}\right) \in E, i<j, v_{i} \notin V C\right\},
\end{aligned}
$$


which satisfy the necessary condition.

$(\Leftarrow)$ : Suppose that $C$ has a normal set basis $B$ containing at most $s=$ $n+4|E|+k$ sets. We can assume without loss of generality that no proper subcollection of $B$ is a normal set basis. We show that $G$ has a vertex cover $V C$ of size at most $k$. Define $V C=\left\{v_{i} \mid\right.$ both $\left\{x_{i}\right\}$ and $\left\{y_{i}\right\}$ are in $\left.B\right\}$. Let $k^{\prime}$ be the number of elements in $V C$. The number of sets in $B$ consisting of only $x_{i}$ and/or $y_{i}$ is at least $n+k^{\prime}$. This can be seen from the fact that $B$ must have the subset $c_{i}$ for all $i$ such that $v_{i} \notin V C$. Thus, there are $n-k^{\prime}$ such sets in addition to $2 k^{\prime}$ singleton sets corresponding to $i$ 's such that $v_{i} \in V C$. Let $E^{\prime} \subseteq E$ be the set of edges covered by $V C$, that is, $E^{\prime}=\left\{\left(v_{i}, v_{j}\right) \mid v_{i}\right.$ or $v_{j}$ is in $\left.V C\right\}$. The following observation can easily be shown (by checking all possibilities):

Observation: For any $e \in E^{\prime}$ at least four sets of $B$ (excluding sets $c_{i}, c_{j}$, $\left\{x_{i}\right\},\left\{y_{i}\right\},\left\{x_{j}\right\}$, or $\left.\left\{x_{j}\right\}\right)$ are necessary to be a normal set basis for the five sets $c_{i j}^{t}, t=1, \ldots, 5$. Further, at least five sets (excluding sets $c_{i}, c_{j},\left\{x_{i}\right\},\left\{y_{i}\right\},\left\{x_{j}\right\}$, or $\left.\left\{x_{j}\right\}\right)$ are required to be a normal set basis for them if $e \notin E^{\prime}$. Notice that, for $e \notin E^{\prime},\left\{x_{i}\right\}$ and $\left\{y_{i}\right\}$, or $\left\{x_{j}\right\}$ and $\left\{y_{j}\right\}$ are never both in $B$, by definition of $E^{\prime}$.

Now the total number of sets needed to cover $C$ is at least $n+k^{\prime}+4\left|E^{\prime}\right|+$ $5\left(|E|-\left|E^{\prime}\right|\right)$, which we know is at most $s=n+4|E|+k$. Hence, we obtain that $n+k^{\prime}+5|E|-\left|E^{\prime}\right| \leq n+4|E|+k$, which implies that $k^{\prime}+|E|-\left|E^{\prime}\right| \leq k$. We conclude the proof by showing that there is a vertex cover $V C^{\prime}$ of size $|E|-\left|E^{\prime}\right|+k^{\prime}$. Add one of the end vertices of each edge $e \in E-E^{\prime}$ to $V C$. This vertex cover is of size $|E|-\left|E^{\prime}\right|+k^{\prime} \leq k$.

The next lemma now follows from the proof of Lemma 4. It defines a set of inputs I for which Normal Set Basis is NP-complete and further shows that for any $(C, s) \in \mathbf{I}$, the collection $C$ has a set basis of size $s$ if and only if $C$ also has a separable normal set basis of size $s$. Of course, the latter property does not hold for the set of all possible inputs for the normal set basis problem.

Lemma 5. There exists a set of inputs I for Normal Set Basis, such that

(1) Normal Set Basis is NP-complete for inputs in I;

(2) for each $(C, s)$ in $\boldsymbol{I}, C$ contains every set at most once and $s<|C|-3$;

(3) for each $(C, s) \in \boldsymbol{I}$, the following are equivalent:

(a) $C$ has a set basis containing s sets.

(b) $C$ has a separable normal set basis containing s sets.

(4) for each $(C, s)$ in $\boldsymbol{I}$, each solution $B$ for $(C, s)$ writes at least two sets of $C$ as a union of at least two sets in $B$.

Proof. The set $\mathbf{I}$ is obtained by applying the reduction in Lemma 4 to inputs $(G, k)$ of Vertex Cover such that $k \leq|E|-3$. This immediately shows (1) and (2). We continue by proving the other cases.

(3) The direction from (b) to (a) is trivial. For the other direction the full proof of Lemma 4 actually shows that if $G$ has a vertex cover of size $k$, then $C$ has a separable normal set basis containing $s$ sets. Conversely, if $C$ has a normal set basis containing at most $s$ sets, then $G$ has a vertex cover of size $k$. This implies that $C$ also has a separable normal set basis containing $s$ sets. 
Hence, we still need to prove that, if $C$ has a set basis of at most $s$ sets, then $C$ also has a normal set basis containing at most $s$ sets. Let $(C, s)$ be an instance in I, i.e., there is an $n \in \mathbb{N}$ and $E \subseteq\{(i, j) \mid 1 \leq i<j \leq n\}$ such that $C=\left\{c_{i} \mid 1 \leq i \leq n\right\} \cup\left\{c_{i j}^{r} \mid(i, j) \in E \wedge 1 \leq r \leq 5\right\}$, and suppose $C$ has a set basis $B=\left\{b_{1}, \ldots, b_{s}\right\}$ of size $s$. We construct a normal set basis for $C$ of size $s$.

To this end, we will show a sequence of assumptions that we can make about $B$ without loss of generality. These assumptions, put together, will imply that $B$ is a normal set basis for $C$, and thus show that if there is a set basis of size $s$, then there is also a normal set basis of size $s$.

Suppose that there is an $i$ such that $B$ contains both $\left\{x_{i}\right\}$ and $\left\{x_{i}, y_{i}\right\}$. Then we can replace $\left\{x_{i}, y_{i}\right\}$ with $\left\{y_{i}\right\}$ and still have a set basis for $C$, since $c_{i}$ is the only set in $C$ of which $\left\{x_{i}, y_{i}\right\}$ is a subset. This gives us our first assumption.

Assumption 1. For every $i \in\{1, \ldots, n\}$, set basis $B$ either contains $\left\{x_{i}, y_{i}\right\}$ or $\left\{x_{i}\right\}$ and $\left\{y_{i}\right\}$, but never both $\left\{x_{i}, y_{i}\right\}$ and $\left\{x_{i}\right\}$ (or, symmetrically, $\left\{x_{i}, y_{i}\right\}$ and $\left.\left\{y_{i}\right\}\right)$.

Suppose there are $1 \leq i<j \leq n$ and $1 \leq r \leq 4$ such that $c_{i j}^{r}$ cannot be formed as a disjoint union of sets from $B$. Without loss of generality, we may assume that $r=1$, i.e., $c_{i j}^{r}=c_{i j}^{1}=\left\{x_{i}, a_{i j}, b_{i j}\right\}$, since all other cases follow by symmetry. Since there are no disjoint sets from $B$ whose union is $c_{i j}^{1}$, there must be two different sets $b^{1}$ and $b^{2}$ in $B$ that are subsets of $c_{i j}$ and contain precisely two elements each. At least one of these subsets must contain $x_{i}$. Assume w.l.o.g. that this set is $b^{1}$. No subset of size two of $c_{i j}^{1}$ that contains $x_{i}$ is a subset of any set of $C$ other than $c_{i j}^{1}$. This means that we can replace $b^{1}$ with $b^{1}-b^{2}$ in $B$ and still have a set basis of size at most $s$, and gives us our second assumption.

Assumption 2. For any $i, j$ and any $r \in\{1, \ldots, 4\}$, the set $c_{i j}^{r}$ can be formed as a union of disjoint sets from $B$.

If $B$ satisfies Assumptions 1 and 2 , but is not normal, there are $1 \leq i<j \leq n$ such that $c_{i j}^{5}$ cannot be formed as a disjoint union of sets from $B$. Let $B_{i j}$ be a subset of $B$ such that the union of the sets in $B_{i j}$ is $c_{i j}^{5}$. We can assume that $B_{i j}$ is inclusion free, i.e., there are no two sets in $B_{i j}$ such that one is a subset of the other, since if there are $b^{1}, b^{2} \in B_{i j}$ with $b^{1} \subset b^{2}$, we can replace $b^{2}$ with $b^{2}-b^{1}$.

Assumption 3. The collection $B_{i j}$ is inclusion free.

If $B_{i j}$ has four members, then we can replace $B_{i j}$ with the four singletons and still have a set basis of size $s$.

Assumption 4. The collection $B_{i j}$ has at most three members.

Suppose there is a set $b$ in $B_{i j}$ such that $b$ is not a subset of any set in $C$ other than $c_{i j}^{5}$. Then we can replace $b$ with $b-\left(\bigcup_{b^{\prime} \in B_{i j}-\{b\}} b^{\prime}\right)$ in $B$ and still have a set basis of size at most $s$. 
Assumption 5. Each member of $B_{i j}$ is a subset of some set from $C$ other that $c_{i j}^{5}$. In particular, this means that each member of $B_{i j}$ has at most two elements.

If we take three different subsets of $c_{i j}^{5}$ with at most two elements, that are also subsets of other sets from $C$ than $c_{i j}^{5}$, then at least two of them are disjoint; see Figure 1. Let these two disjoint sets be $b^{1}$ and $b^{2}$. If $b^{1}$ and $b^{2}$ both contain two elements, we can replace $B_{i j}$ with $\left\{b^{1}, b^{2}\right\}$.

Assumption 6. There are at most two sets in $B_{i j}$ with two elements. Furthermore, these two sets must overlap. This means that $B_{i j}$ must have exactly three members, two with two elements and one singleton. Without loss of generality, we may assume that $B_{i j}=\left\{\left\{a_{i j}, b_{i j}\right\},\left\{b_{i j}, d_{i j}\right\},\left\{e_{i j}\right\}\right\}$. (All other cases are symmetrical.) We may also assume that neither $\left\{a_{i j}\right\}$ nor $\left\{b_{i j}\right\}$ belong to $B$. (If $\left\{a_{i j}\right\}$ belongs to $B$ then we can replace $\left\{a_{i j}, b_{i j}\right\}$ by $\left\{b_{i j}\right\}$ in $B_{i j}$, and if $\left\{b_{i j}\right\}$ belongs to $B$ we can replace $\left\{b_{i j}, d_{i j}\right\}$ by $\left\{d_{i j}\right\}$ in $B_{i j}$.)

If $B_{i j}$ satisfies Assumption 6, in order to form $c_{i j}^{1}$ either $\left\{x_{i}\right\},\left\{x_{i}, a_{i j}\right\}$, $\left\{x_{i}, b_{i j}\right\}$, or $\left\{x_{i}, a_{i j}, b_{i j}\right\}$ must be a member of $B$. If it is not $\left\{x_{i}\right\}$, we can replace it with $\left\{x_{i}\right\}$, since none of the other sets is a subset of any other set in $C$ than $c_{i j}^{1}$. But if $\left\{x_{i}\right\} \in B$ we can also assume that $\left\{y_{i}\right\} \in B$. To form $c_{i j}^{3}, B$ must, apart from $\left\{y_{i}\right\}$ and $\left\{e_{i j}\right\}$, contain some subset of $c_{i j}^{3}$ that contains $d_{i j}$. Since we have both $\left\{y_{i}\right\}$ and $\left\{e_{i j}\right\}$ in $B$, we may replace this subset with $\left\{d_{i j}\right\}$. Once we have $\left\{d_{i j}\right\}$ in $B$, we can replace $\left\{b_{i j}, d_{i j}\right\}$ by $\left\{b_{i j}\right\}$ in $B$.

Assumption 7. There are disjoint members of $B$ whose union is $c_{i j}^{5}$.

Together, Assumptions 1,2, and 7 imply that $B$ is in fact a normal set basis for $C$. Since each assumption was made without loss of generality, we have shown that from any set basis for $C$ of size $s$ we can form a normal set basis for $C$ of size at most $s$.

(4) We simply observe that a normal set basis writing at most one set of $C$ as a union of at least two sets must contain at least $|C|$ sets, and hence cannot be a solution for $(C, s)$, since $s<|C|$.

The proof of the following lemma is partly inspired by the proof of Theorem 3.1 of [11], but we significantly strengthen it for our purposes.

Lemma 6. There exists a set of regular languages $\mathcal{L}$ such that

(1) DFA $\rightarrow \delta N F A$ minimization is NP-complete for DFAs accepting $\mathcal{L}$ and

(2) for each $L \in \mathcal{L}$, the size of the minimal $N F A$ for $L$ is equal to the size of the minimal $\delta N F A$ for $L$, minus 1.

Proof. The NP upper bound is immediate, as equivalence testing for unambiguous finite automata is in PTIME [17]. We can guess a $\delta$ NFA of sufficiently small size and test in PTIME whether it is equivalent to the given DFA. 
For the lower bound, we reduce from Separable Normal Set Basis. To this end, let $(C, s)$ be an input of Separable Normal Set Basis. Hence, $C$ is a collection of $n$ sets and $s$ is an integer. According to Lemma 5, we can assume without loss of generality that $(C, s) \in \mathbf{I}$, that is, $C$ has a separable normal set basis containing $s$ sets if and only if $C$ has a normal set basis of size $s$. Moreover, we can assume that $s<n-3$.

We construct in LOGSPACE a DFA $A$ and an integer $\ell$ such that the following are equivalent:

- $C$ has a separable normal set basis of size at most $s$.

- There exists a $\delta$ NFA $N_{\delta}$ for $L(A)$ of size at most $\ell$.

- There exists an NFA $N$ for $L(A)$ of size at most $\ell-1$.

The DFA $A$ accepts the language $\{a c b \mid c \in C$ and $b \in c\}$, which is a finite language of strings of length three.

Formally, let $C=\left\{c_{1}, \ldots, c_{n}\right\}$ and $c_{i}=\left\{b_{i, 1}, \ldots, b_{i, n_{i}}\right\}$. Then, $A$ is defined over $\operatorname{Alpha}(A)=\{a\} \cup \bigcup_{1<i<n}\left\{c_{i}, b_{i, 1}, \ldots, b_{i, n_{i}}\right\}$. The state set of $A$ is $\operatorname{States}(A)=\left\{q_{0}, q_{0}^{\prime}, q_{1}, \ldots, q_{n}, q_{f}\right\}$, and the initial and final state sets of $A$ are $q_{0}$ and $q_{f}$, respectively. The transitions $\operatorname{Rules}(A)$ are formally defined as follows:

$-q_{0} \stackrel{a}{\rightarrow} q_{0}^{\prime}$

- for every $i=1, \ldots, n, q_{0}^{\prime} \stackrel{c_{i}}{\rightarrow} q_{i}$; and

- for every $i=1, \ldots, n$ and $j=1, \ldots, n_{i}, q_{i} \stackrel{b_{i, j}}{\longrightarrow} q_{f}$.

Finally, define $\ell:=s+4$.

Obviously, $A$ and $\ell$ can be constructed from $C$ and $s$ using logarithmic space. Observe that due to Lemma $5, C$ contains every set at most once, and hence does not contain $c_{i}=c_{j}$ with $i \neq j$. Hence, $A$ is a minimal DFA for $L(A)$.

We now show that,

(a) if $C$ has a separable normal set basis containing at most $s$ sets, then there exists a $\delta$ NFA $N_{\delta}$ for $L(A)$ of size at most $\ell$ and an NFA $N$ for $L(A)$ of size at most $\ell-1$;

(b) if there exists a $\delta \mathrm{NFA} N_{\delta}$ for $L(A)$ of size at most $\ell$ then $C$ has a separable normal set basis containing at most $s$ sets; and

(c) if there exists an NFA $N$ for $L(A)$ of size at most $\ell-1$ then $C$ has a separable normal set basis containing at most $s$ sets.

(a) Assume that $C$ has a separable normal set basis containing $s$ sets. We construct a $\delta \mathrm{NFA} N_{\delta}$ for $L(A)$ of size $\ell=s+4$.

Let $B=\left\{r_{1}, \ldots, r_{s}\right\}$ be the separable normal set basis for $C$ containing $s$ sets. Also, let $B_{1}$ and $B_{2}$ be disjoint subcollections of $B$ such that each element of $C$ is either an element of $B_{1}$, an element of $B_{2}$, or a disjoint union of an element of $B_{1}$ and an element of $B_{2}$.

To describe $N_{\delta}$, we first fix the representation of each set $c$ in $C$ as a disjoint union of at most one set in $B_{1}$ and at most one set in $B_{2}$. Say that each basic member of $B$ in this representation belongs to $c$. 
We define the state set of $N_{\delta}$ as $\operatorname{States}\left(N_{\delta}\right)=\left\{q_{0}, q_{1}, q_{2}, q_{f}\right\} \cup\left\{r_{i} \in B_{1}\right\} \cup\left\{r_{i} \in\right.$ $\left.B_{2}\right\}$. The transition rules of $N_{\delta}$ are defined as follows. First, Rules $\left(N_{\delta}\right)$ contains the non-deterministic transitions $q_{0} \stackrel{a}{\rightarrow} q_{1}$ and $q_{0} \stackrel{a}{\rightarrow} q_{2}$. Furthermore, for every $i=1, \ldots, n, j=1, \ldots, s$, and $m=1,2$, Rules $\left(N_{\delta}\right)$ contains the rule

$-q_{m} \stackrel{c_{i}}{\rightarrow} r_{j}$, if $r_{j} \in B_{m}$ and $r_{j}$ belongs to $c_{i}$; and

$-r_{j} \stackrel{b}{\rightarrow} q_{f}$, if $b \in r_{j}$.

Notice that the size of $N_{\delta}$ is $|B|+4=s+4=\ell$. By construction, we have that $L\left(N_{\delta}\right)=L(A)$.

We argue that $N_{\delta}$ is a $\delta$ NFA. First, we argue that the transitions $q_{0} \stackrel{a}{\rightarrow} q_{1}$ and $q_{0} \stackrel{a}{\rightarrow} q_{2}$ are the only non-deterministic transitions. By construction, all transitions going to $q_{f}$ are deterministic. It is also easy to see that all outgoing transitions from $q_{1}$ are deterministic because, if we assume that $N_{\delta}$ contains transitions of the form $q_{1} \stackrel{c_{i}}{\rightarrow} r_{j}$ and $q_{1} \stackrel{c_{i}}{\rightarrow} r_{j^{\prime}}$ with $j \neq j^{\prime}$, this would mean that both $r_{j}$ and $r_{j^{\prime}}$ belong to $c_{i}$, which contradicts the definition of $B_{1}$. The argument for $q_{2}$ is analogous.

Next, we show that $N_{\delta}$ is unambiguous. Towards a contradiction, assume that the string $a c_{i} b$ has two accepting runs. The only way in which this can happen is that one run visits state $q_{1}$ and the other run visits state $q_{2}$. Let $r_{j_{1}}$ (respectively, $r_{j_{2}}$ ) be the state such that $q_{1} \stackrel{c_{i}}{\rightarrow} r_{j_{1}}$ (respectively, $q_{2} \stackrel{c_{i}}{\rightarrow} r_{j_{2}}$ ) are transitions in $N_{\delta}$. By construction of $N_{\delta}$, we have that $j_{1} \neq j_{2}$. But this means that both $r_{j_{1}}$ and $r_{j_{2}}$ belong to $c_{i}$, and their intersection contains the element $b$, which contradicts the disjointness condition of the normal set basis $B$.

Finally, the NFA $N$ for $L(A)$ is obtained by merging the two states $q_{1}$ and $q_{2}$ from $N_{\delta}$. Obviously, the size of $N$ is $\ell-1$.

(b) Assume that $L(A)$ can be accepted by a $\delta \mathrm{NFA} N_{\delta}$ of size at most $\ell$. We can assume that $N_{\delta}$ is minimal. We need to show that there exists a separable normal set basis for $C$ containing at most $s=\ell-4$ sets.

Recall that we assumed that $s<n-3$. Hence, we have that $\ell=s+4<$ $n+1=|A|-1$. As we observed that $A$ is a minimal DFA for $L(A)$, it must be the case that $N_{\delta}$ has at least one non-deterministic transition.

Notice that, as $N_{\delta}$ is minimal and accepts only strings of length three, we can assume that $N_{\delta}$ has a unique final state. Let $q_{0}$ and $q_{f}$ be the initial and final state of $N_{\delta}$, respectively. Furthermore, we can partition $N_{\delta}$ 's states into four sets $\mathcal{Q}_{0}, \mathcal{Q}_{1}, \mathcal{Q}_{2}$, and $\mathcal{Q}_{3}$ such that, for each $0 \leq i \leq 3, \mathcal{Q}_{i}$ is precisely the set of states that $N_{\delta}$ can be in after having read a string of length $i$. For each state $q \in \mathcal{Q}_{2}$, define a set $B_{q}=\left\{b \mid q \stackrel{b}{\rightarrow} q_{f} \in \operatorname{Rules}\left(N_{\delta}\right)\right\}$.

Next, we show that the collection $B=\left\{B_{q} \mid q \in \mathcal{Q}_{2}\right\}$ is a normal set basis for $C$. By definition of $L(A)$, we have that every $c \in C$ is the union of $B_{c}:=\left\{B_{q} \mid \exists p \in \mathcal{Q}_{1}: p \stackrel{c}{\rightarrow} q \in \operatorname{Rules}\left(M_{i}\right)\right\}$. It remains to show that $B_{c}$ is also a disjoint subcollection of $B$. When $B_{c}$ contains only one set, there is nothing to prove. Towards a contradiction, assume that $B_{c}$ contains two different sets $B_{q_{1}}$ and $B_{q_{2}}$ such that $a \in B_{q_{1}} \cap B_{q_{2}}$. By definition of $B$, this would mean that the string $a c b$ has two accepting runs: $q q_{1} q_{f}$ and $q q_{2} q_{f}$ with $q_{1} \neq q_{2}$. But as $N_{\delta}$ is unambiguous, this is impossible. Hence, $B_{c}$ is a disjoint subcollection of $B$. 
Finally, we want to prove that $B$ contains at most $\ell-4$ sets. Notice that $B$ contains at most $\ell-4$ sets if and only if $\mathcal{Q}_{1}$ contains at least two states. Towards a contradiction, assume that $\mathcal{Q}_{1}$ is a singleton $q$. By Lemma 5, we know that there are at least two sets $c_{1}, c_{2}$ such that $B_{c_{1}}$ and $B_{c_{2}}$ contain two sets. In other words, there are at least two alphabet symbols $c_{1}$ and $c_{2}$ that have nondeterministic outgoing transitions from $q$, which contradicts that $N_{\delta}$ is a $\delta \mathrm{NFA}$. Hence, $B$ is a normal set basis for $C$ of size at most $\ell-4$. As $(C, s) \in \mathbf{I}$, we have that $C$ has a separable normal set basis of size $s=\ell-4$ by Lemma 5 .

(c) Let $N$ be an NFA for $L(A)$ of size at most $\ell-1$. We can assume that $N$ is minimal. We will show that there exists a set basis for $C$ containing at most $s=\ell-4$ sets.

Recall that we assumed that $s<n-3$. Hence, we have that $\ell=s+4<$ $n+1=|A|-1$. As we observed that $A$ is a minimal DFA for $L(A)$, it must be the case that $N$ has at least one non-deterministic transition.

Notice that, as $N$ is minimal and accepts only strings of length three, we can assume that $N$ has a unique final state. Let $q_{0}$ and $q_{f}$ be the initial and final state of $N$, respectively. Furthermore, we can partition $N$ 's states into four sets $\mathcal{Q}_{0}, \mathcal{Q}_{1}, \mathcal{Q}_{2}$, and $\mathcal{Q}_{3}$ such that, for each $0 \leq i \leq 3, \mathcal{Q}_{i}$ is precisely the set of states that $N$ can be in after having read a string of length $i$. For each state $q \in \mathcal{Q}_{2}$, define a set $B_{q}=\left\{b \mid q \stackrel{b}{\rightarrow} q_{f} \in \operatorname{Rules}\left(N_{\delta}\right)\right\}$. Next, we show that the collection $B=\left\{B_{q} \mid q \in \mathcal{Q}_{2}\right\}$ is a set basis for $C$. By definition of $L(A)$, we have that every $c \in C$ is the union of $B_{c}:=\left\{B_{q} \mid \exists p \in \mathcal{Q}_{1}: p \stackrel{c}{\rightarrow} q \in \operatorname{Rules}\left(M_{i}\right)\right\}$. As $\mathcal{Q}_{0}, \mathcal{Q}_{1}$, and $\mathcal{Q}_{3}$ contain at least one state, we also have that $B$ contains at most $\ell-1-3=s$ sets.

From Lemma 5 , it now follows that $C$ also has a separable normal set basis containing at most $s$ sets.

We are now ready to finish the proof of Theorem 2 .

Proof of Theorem 2. Let $\mathcal{N}$ be a class of finite automata. If $\delta N F A \subseteq \mathcal{N}$ then $D F A \rightarrow \mathcal{N}$ minimization is NP-hard.

Proof. In this Section we provided a reduction from Vertex Cover to DFA $\rightarrow$ $\delta$ NFA minimization, and showed that, for the regular languages we consider, the minimal NFA is 1 state smaller than the minimal $\delta \mathrm{NFA}$.

Let $\mathcal{N}$ be a class of finite automata such that $\delta$ NFA $\subseteq \mathcal{N} \subseteq$ NFA. As was shown in the proof of Lemma 6 , any decision algorithm for DFA $\rightarrow \mathcal{N}$ minimization can approximate the DFA $\rightarrow \delta$ NFA minimization problem within a term 1 (as the minimal NFA is only one state smaller than the minimal $\delta \mathrm{NFA}$ ).

As can be seen from the other proofs in Section 3, this approximation algorithm for DFA $\rightarrow \delta$ NFA minimization can easily be adapted to an approximation algorithm for Vertex Cover within a term 1. As we know that it is NP-hard to approximate Vertex Cover within a constant term, we can conclude that DFA $\rightarrow \mathcal{N}$ minimization is also NP-hard.

Until now, our results focused on classes of finite automata that can accept all regular languages. Our proof shows that this is not even necessary, as the 
NP-hard instances we construct only accept strings of length tree. Therefore, we also have the following Corollary.

Corollary 7. Let $\delta N F A_{\text {finite }}$ be the class of $\delta N F A$ s that accept only finite languages. Let $\mathcal{N}$ be class of finite automata. If $\delta N F A_{\text {finite }} \subseteq \mathcal{N}$ then the DFA $\rightarrow \mathcal{N}$ minimization problem is NP-hard.

\section{Succinctness and Uniqueness}

As mentioned in the introduction, when a developer selects a description mechanism for regular languages, she faces a trade-off between succinctness and complexity of minimization. The following proposition shows that in the case of $\delta \mathrm{NFAs}$, the succinctness bought at the price of NP-completeness is limited.

Proposition 8. For every $\delta N F A$ of size $n$, there is an equivalent DFA of size $O\left(n^{2}\right)$.

Proof. For every finite automaton that can choose non-deterministically between two different start states but is otherwise deterministic (a 2-MDFA), there is an equivalent DFA with at most quadratically many states [7].

Let $A$ be a $\delta$ NFA, and let $(q, a)$ be the only pair in $\operatorname{States}(A) \times \operatorname{Alpha}(A)$ such that degree $(q, a)=2$. Let $q_{1}$ and $q_{2}$ be the two states reachable from $q$ when reading an $a$. If we remove all states from $A$ that are reachable neither from $q_{1}$ nor from $q_{2}$, and make $q_{1}$ and $q_{2}$ initial states, we obtain a 2-MDFA $A^{\prime}$. We can compute the smallest DFA $A^{\prime \prime}$ equivalent to $A^{\prime}$ in quadratic time. Now, all we need to do is to add $A^{\prime \prime}$ to $A$ and replace the two rules going from $q$ to $q_{1}$ and $q_{2}$, respectively, by a single rule that reads an $a$ and goes to the initial state of $A^{\prime \prime}$. The size of the new, deterministic, automaton is $|\operatorname{States}(A)|+\left|\operatorname{States}\left(A^{\prime \prime}\right)\right|=$ $O\left(|\operatorname{States}(A)|^{2}\right)$.

On the other hand, if we were to remove the $\operatorname{branch}(A) \leq 2$ condition in the definition of $\delta \mathrm{NFAs}$, then there would be an exponential gain in succinctness. This is witnessed by the standard family of languages $(a+b)^{*} a(a+b)^{n}$ for $n \geq 0$ that shows that NFAs are exponentially more succinct than DFAs in general. The canonical NFA for this language is unambiguous and has only one pair $(q, a)$ for which degree $(q, a)=2$.

Proposition 9. The minimal $\delta N F A$ for a regular language is not unique.

Proof. Consider the language $L$ defined by the regular expression

$$
r=(a+b) a a a+b(a+b)(a+b) b .
$$

Figure 2 depicts two $\delta$ NFAs, $A$ and $B$, that both accept $L$. We argue that eight is the minimal number of states for any $\delta$ NFA that accepts $L$. First, it is clear that any such automaton has to remember how many letters it has read so far. Second, the automaton has to have at least two states that can be reached after 
reading one letter, one that accepts the string $b b b$ and one that does not. Third, there must also be at least two states reachable after reading two letters, one that accepts the string $b b$ and one that doesn't. Fourth, there must be at least two states that can be reached after reading three letters, one that accepts the string $b$ and one that does not. Together with the fact that there has to be one initial state and at least one final state, this shows that any $\delta \mathrm{NFA}$ for $L$ needs at least eight states.

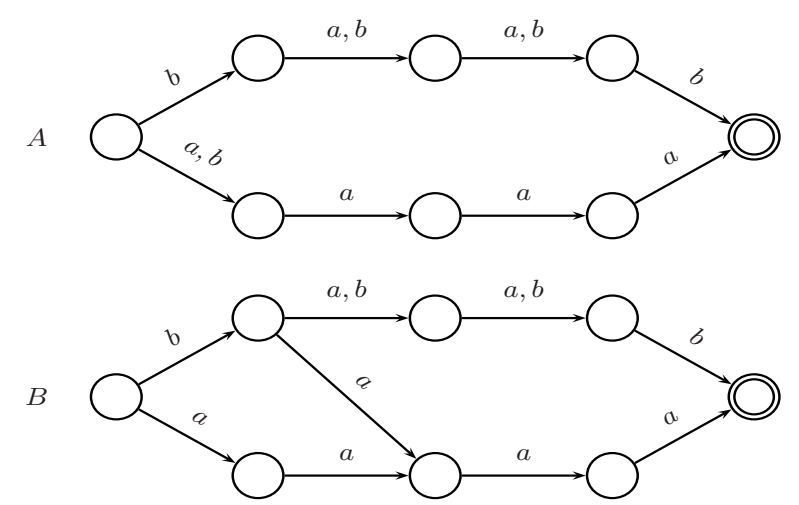

Fig. 2. Two minimal $\delta$ NFAs, that both accept the language $L$ from the proof of Proposition 9 .

\section{Automata with Multiple Initial States}

Throughout the paper, to simplify definitions, we have assumed that finite automata have a unique start state. As we mentioned in the Introduction, the minimization problem for finite automata that can non-deterministically choose between multiple initial states, but are otherwise deterministic, has also been studied $[7,12]$.

Proposition 10. Minimization is NP-hard for unambiguous finite automata that have at most two initial states but are otherwise deterministic.

Proof. We simply have to reconsider the proof of Lemma 6, change $L(A)$ so that it accepts the $c b$ suffixes of its current language, consider the $\delta$ NFA we construct without its start state, and make the two successors of the start state initial states (and analogously for the NFA considered in the proof). The rest of the proof carries through virtually unchanged.

Together with Theorem 2 this answers all the open questions mentioned by Malcher [12]. 


\section{References}

1. P. Abdulla, J. Deneux, L. Kaati, and M. Nilsson. Minimization of non-deterministic automata with large alphabets. In CIAA, pages 31-42, 2006.

2. J. Goldstine, M. Kappes, C Kintala, H. Leung, A. Malcher, and D. Wotschke. Descriptional complexity of machines with limited resources. J. Univ. Comp. Science, 8(2):193-234, 2002.

3. G. Gramlich and G. Schnitger. Minimizing NFAs and regular expressions. JCSS, 73(6):908-923, 2007.

4. H. Gruber and M. Holzer. Finding lower bounds for nondeterministic state complexity is hard. In $D L T$, pages 363-374, 2006.

5. H. Gruber and M. Holzer. Computational complexity of NFA minimization for finite and unary languages. In LATA, pages 261-272, 2007.

6. H. Gruber and M. Holzer. Inapproximability of nondeterministic state and transition complexity assuming $\mathrm{P} \neq \mathrm{NP}$. In $D L T$, pages $205-216,2007$.

7. M. Holzer, K. Salomaa, and S. Yu. On the state complexity of $k$-entry deterministic finite automata. J. Automata, Languages, and Combinatorics, 6(4):453-466, 2001.

8. J. Hromkovic, J. Karhumäki, H. Klauck, G. Schnitger, and S. Seibert. Measures of nondeterminism in finite automata. In ICALP, pages 199-210, 2000.

9. J. Hromkovic and G. Schnitger. Comparing the size of NFAs with and without epsilon-transitions. TCS, 380(2):100-114, 2007.

10. T. Jiang, E. McDowell, and B. Ravikumar. The structure and complexity of minimal NFAs over unary alphabet. Int. J. Found. Comp. Science, 2:163-182, 1991.

11. T. Jiang and B. Ravikumar. Minimal NFA problems are hard. Siam J. Comp., 22(6):1117-1141, 1993.

12. A. Malcher. Minimizing finite automata is computationally hard. TCS, 327(3):375390, 2004.

13. A. Meyer and M.J. Fischer. Economy of descriptions by automata, grammars, and formal systems. In FOCS, pages 188-191. IEEE, 1971.

14. R Paige and R. Tarjan. Three parition refinement algorithms. Siam J. Comp., 16:973-989, 1987.

15. K. Salomaa. Descriptional complexity of nondeterministic finite automata. In DLT, pages 31-35, 2007.

16. G. Schnitger. Regular expressions and NFAs without epsilon-transitions. In STACS, pages 432-443, 2006.

17. R. E. Stearns and H. B. Hunt III. On the equivalence and containment problems for unambiguous regular expressions, regular grammars and finite automata. Siam J. Comp., 14(3):598-611, 1985.

18. L. Stockmeyer and A. Meyer. Word problems requiring exponential time: Preliminary report. In STOC, pages 1-9. ACM, 1973. 